Diabetologia 11, 105-111 (1975)

๑ by Springer-Verlag 1975

\title{
Antifibrin Action of Phenformin
}

\author{
R. N. Banerjee, V. Kumar, S. R. Rao, A. L. Sahni, M. Arya and J. Bardhan \\ Dept. of Haematology and Nuclear Medicine and Dept. of Medicine, Safdarjang Hospital, New Delhi, India
}

Received: July 9, 1974, and in revised form: December 5, 1974

\begin{abstract}
Summary. Effects of phenformin on blood sugar, serum triglyceride, thrombin time, euglobulin clot lysis time and cardiovascular complications were studied in maturity onset diabetics and in atherosclerotic patients with or without diabetes, for a period of 14-18 months. Phenformin has shown the characteristic properties of an antifibrinopathic agent in that it prolongs thrombin time and enhances fibrinolysis. The hypoglycaemic effect of phenformin was found to be directly related to its antifibrinopathic action. Plasma lipids fell in all cases. Absence of fresh cardiovascular complications and improvement in anginal symptoms were observed. The metabolic, haematological and clinical benefits of phenformin and its limitations in maturity onset diabetes
\end{abstract}

and atherosclerosis may be explained by the effects of the drug upon the thrombin-fibrinogen reaction. These results lend support to the hypothesis of a primary fibrinopathic pathogenesis in maturity onset diabetes mellitus and atherosclerosis.

Key words: Thrombin time, thrombin-fibrinogen reaction, fibrinolytic activity, plasminogen activator, phenformin, antifibrinopathic action, lipid lowering effect, hypoglycaemic effect, plasma insulin clearance, serum triglyceride, euglobulin clot lysis time, inter-relationship between hypoglycaemic and antifibrinopathic effect, slow consumptive fibrinocoagulopathy, fibrinocoagulopathic pathogenesis.
Intravascular coagulation due to an intrinsic Antithrombin III deficiency has been reported in both maturity onset diabetes mellitus and atherosclerosis $[1,2,3,4]$. It has been suggested that in maturity onset diabetes mellitus this leads to an intravascular fibrin silting' in the microvascular bed, presenting a pathological barrier to plasma insulin clearance, resulting in hyperinsulinaemia, hyperglycaemia and hyperlipaemia [3]. Experimental support for this hypothesis has recently been provided by the production of a fibrinopathic diabetic state in animals [5]. A corollary to this hypothesis is that the clinical and metabolic changes seen in the two disorders (diabetes and atherosclerosis) should be reversible by an appropriate agent which would retard the formation and enhance dissolution of the intravascular fibrin silting. In recent years phenformin hydrochloride has been reported to have significant fibrinolytic activity $[6,7$, $8,9,10]$, though the mechanism of action is not known. The present work was undertaken to investigate the possible antifibrin actions of phenformin, by studying its effect on thrombin time and fibrinolysis, and also to examine the validity of the original hypothesis by studying metabolic and haematological effects of the drug in the two disorders.

\section{Materials and Methods}

The action of phenformin was examined in vivo and in vitro.

\section{In Vivo Studies}

Fifty seven patients with maturity onset diabetes and/or atherosclerosis (myocardial infarction and angina pectoris), aged 35 to 65 years, were studied for a period of 14 to 18 months. The diagnosis in each case was established by clinical examination, fundoscopy, electrocardiogram, urinalysis and blood biochemistry. On the basis of the examinations and a $100 \mathrm{gm}$ oral glucose tolerance test, the patients were divided into four clinical groups. The criteria for diagnosing diabetes were those of Fajans and Conn [11].

\section{Clinical Groups}

Group A. Maturity onset diabetes mellitus without detectable atherosclerosis - 25 cases.

Group B. Maturity onset diabetes mellitus with atherosclerosis -10 cases.

Group C. Non-diabetic atherosclerosis -10 cases.

Group $D$. Atherosclerotic subjects found to be diabetic -12 cases.

\section{Treatment Schedule}

All patients included in the study received 75 to $100 \mathrm{mg}$ phenformin (DBI, U.S. Vitamin Corp) in divided doses, either a plain tablet or a sustained release capsule, for a period of 14-18 months. Diabetic patients were kept on an 1800-2000 calorie diet during the treatment. Patients requiring supple- 
mentary insulin or oral sulphonylureas, were excluded from the study.

\section{Follow Up}

All patients were seen in the Research Clinic once a month for the first two visits; thereafter they were examined every two months for the period of the study.

\section{Methods}

The following blood assays were carried out:

1. Blood sugar estimation was carried out by the method of Asatoor and King [12]. During the follow up studies, one and a half hour post prandial blood samples were assayed.

2. Serum triglyceride estimations were done before and after 4 months' of treatment by the method of Van Handel and Zilversmit [13].

3. Thrombin time was determined by the method described in our earlier communications $[1,3]$.

4. Euglobulin clot lysis time (ECLT) was measured before and at the end of 3 and 6 months of treatment by the method of Von Kaulla [14].

\section{In Vitro Study}

The in vitro fibrinolytic effect of phenformin was studied in the plasma samples from normal and untreated subjects with maturity onset diabetes mellitus and atherosclerosis. Simultaneous determinations of euglobulin clot lysis time on each sample were carried out with three coded buffer solutions viz., (a) barbitone buffer, $\mathrm{pH} 7.3$ (b) one mgm per cent phenformin in barbitone buffer and (c) $5 \mathrm{mgm}$ per cent phenformin in barbitone buffer. The phenformin was dissolved in barbitone buffer by boiling for 2 to $3 \mathrm{~min}$. The $5 \mathrm{mgm}$ per cent solution gave a light milky appearance without any deposit on standing. The final $\mathrm{pH}$ of the solutions was adjusted at $\mathrm{pH}$ 7.3.

\section{Trial Method}

The study was carried out double blind by four independent teams of clinicians and laboratory workers. The two clinical teams made their independent assessment on alternate visits. The two laboratory teams carried out the metabolic (blood sugar and serum triglyceride) and haematological (thrombin time and ECLT) investigations independently. The final analysis of the results was made at the end of the study.
Statistical Analysis

The statistical significance of the laboratory data was evaluated by Student's ' $t$ ' test for unpaired data by the Biostatistics Division of Indian Council of Medical Research.

\section{Results}

\section{In Vivo Study}

\section{Clinical Observations}

No death occurred in any of the observation groups. No evidence of fresh cardiovascular complications was observed in the diabetics of groups A, $B$ and D. In group C, all patients continued to be euglycaemic and only one subject with a previous myocardial infarction developed a mild ischaemic attack, with transient E.C.G. changes, after six months of treatment. The patient made an uneventful recovery and remained asymptomatic thereafter. In twenty cases with angina pectoris, belonging to the groups $\mathrm{B}, \mathrm{C}$ and $\mathrm{D}$, a remarkable improvement was noted. The disappearance of the angina and increased exercise tolerance led to the discontinuation of so called coronary dilators.

\section{Haematological Effects}

(i) Thrombin time (TT): The mean values of TT in all the four observation groups are shown in figure $\mathrm{I}$. A reduced TT (mean 63 seconds, control $83 \pm 12.25$ ) was observed in all patients before treatment. In all the groups a very highly significant $(P<0.001)$ prolongation of the TT was observed from the second month; thereafter, all the patients maintained a normal TT during the entire period of study.

(ii) Euglobulin clot lysis time (ECLT): The mean ECLT values in groups (A\&B) and (C\&D) up to six months of treatment are shown in Table 1 . In all cases, a reduced plasma fibrinolytic activity, as measured by prolonged ECLT (mean $263.0 \mathrm{~min}$, control $113 \mathrm{~min}$ ) was observed before treatment. A very highly significant reduction in the ECLT values $(P<0.001)$ with treatment was observed in all the groups. The values at the end of six months of treatment (194.06 and $180.82 \mathrm{~min}$ ), however, were still significantly higher than normal, indicating the persistence of reduced fibrinolytis.

\section{Metabolic Effects}

(i) Blood sugar: The mean values of one and half hour blood samples in the four observation groups 

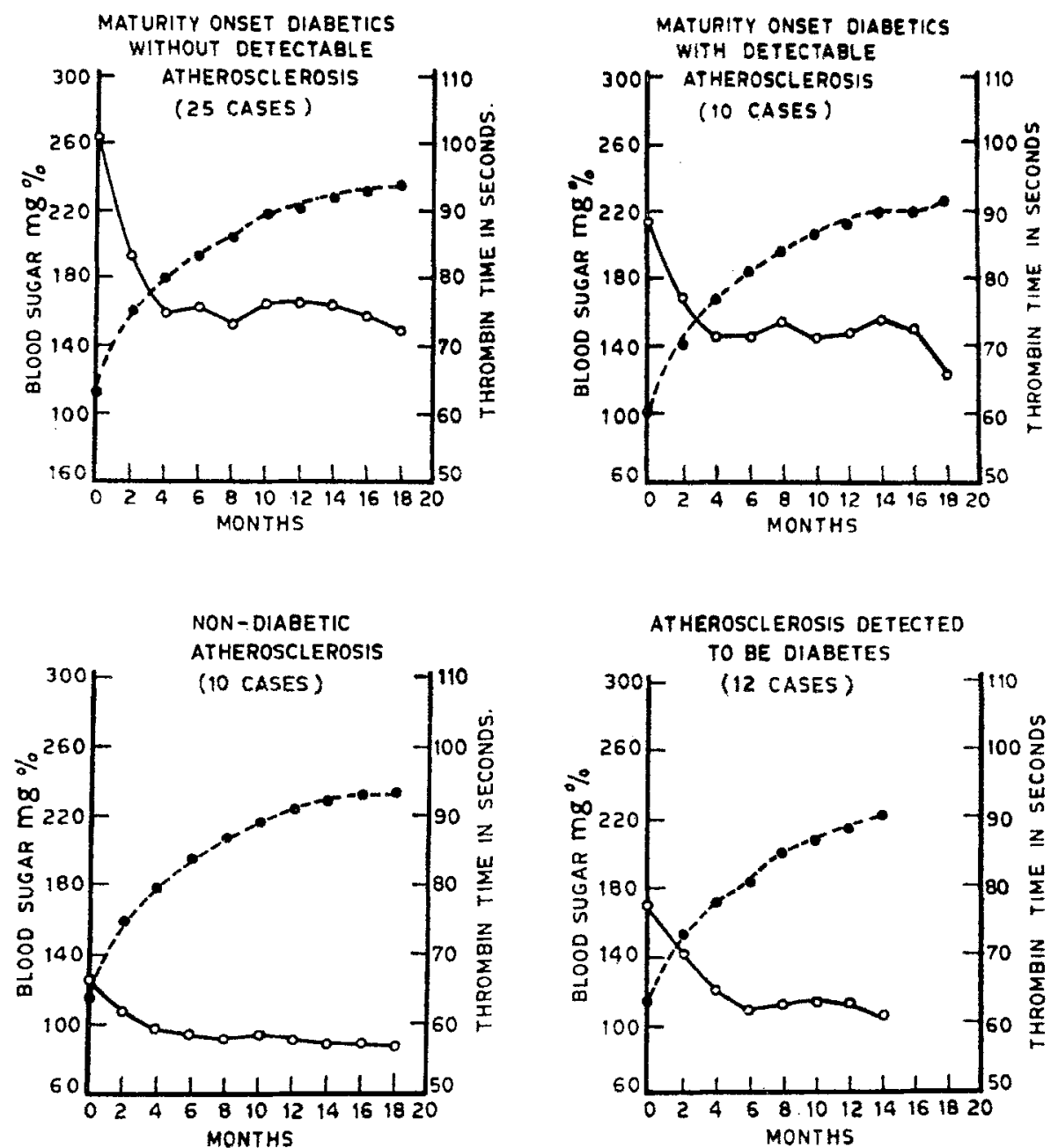

Fig. 1. In-vivo effects of phenformin on thrombin time (-groups of diabetes and atherosclerosis

Table 1. Effect of phenformin on serum triglyceride and euglobulin clot lysis time in (A) Maturity onset diabetes without atherosclerosis. (B) Maturity onset diabetes with atherosclerosis. (C) Non-diabetic atherosclerosis and (D) Atherosclerosis found to be diabetic

\begin{tabular}{|c|c|c|c|c|c|c|c|c|}
\hline \multirow{2}{*}{$\begin{array}{l}\text { Obser- } \\
\text { vation } \\
\text { group }\end{array}$} & \multirow{2}{*}{$\begin{array}{l}\text { No. of } \\
\text { cases }\end{array}$} & \multicolumn{3}{|c|}{$\begin{array}{l}\text { Mean serum triglyceride } \\
\text { (mg per cent) }\end{array}$} & \multicolumn{4}{|c|}{$\begin{array}{l}\text { Mean euglobulin clot lysis time } \\
\text { (Minutes) }\end{array}$} \\
\hline & & $\begin{array}{l}\text { Before } \\
\text { treat- } \\
\text { ment }\end{array}$ & $\begin{array}{l}\text { After } 4 \\
\text { months }\end{array}$ & $\mathbf{P}$ & $\begin{array}{l}\text { Before } \\
\text { treat- } \\
\text { ment }\end{array}$ & $\begin{array}{l}\text { After } 3 \\
\text { months }\end{array}$ & $\begin{array}{l}\text { After } 6 \\
\text { months }\end{array}$ & $P$ \\
\hline A & 25 & 68.88 & 59.92 & $<0.001$ & 272.74 & 233.88 & 194.06 & $<0.001$ \\
\hline B & 10 & 68.70 & 59.70 & $<0.001$ & & & & \\
\hline C & 10 & 65.90 & 56.30 & $<0.001$ & 253.32 & 211.82 & 180.82 & $<0.001$ \\
\hline D & 12 & 66.56 & 56.32 & $<0.001$ & & & & \\
\hline
\end{tabular}


are shown in Fig. 1. In all the diabetic cases (Groups A, B and D) a progressive reduction of hyperglycaemia was observed. In the non-diabetic atherosclerotics (group C), the blood sugar level remained within the normal range and no patient developed hypoglycaemia, though the mean level fell.

(ii) Serum triglyceride: The results are shown in Table 1 . In all the groups, a very highly significant $(P<0.001)$ reduction was observed with phenformin therapy.

(iii) Correlations between phenformin therapy, blood sugar and thrombin time changes are shown in Fig. 2. In all the four observation groups, the reduction in the blood sugar level was found to be directly related to the increase in the thrombin time, till the thrombin time attained the normal range of 85 to $100 \mathrm{sec}$. Thereafter, with the normal thrombin time, there was no further reduction in the blood sugar despite continued phenformin therapy.
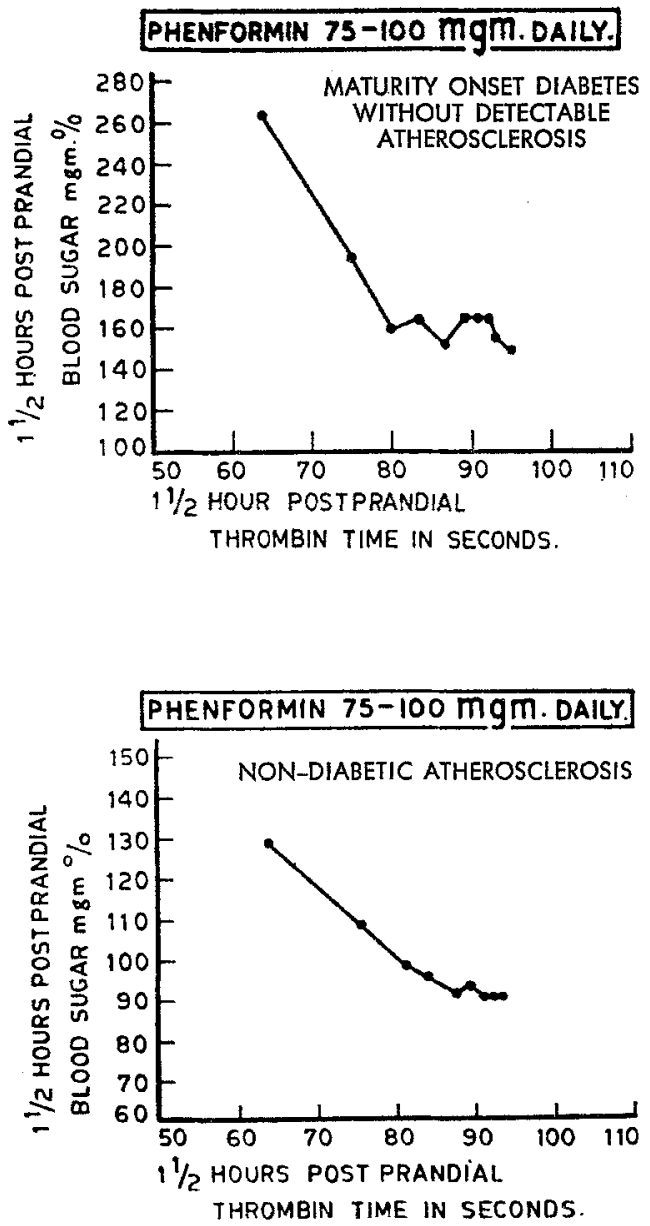

\section{In Vitro Fibrinolytic Activity of Phenformin}

The mean values of ECLT in plasma from seven normal, eleven diabetic and twelve atherosclerotic patients, as determined by the three coded buffer solutions, are shown in Fig. 3. A highly significant $(P<0.001)$ reduction in the ECLT was observed in all the plasma samples with $1 \mathrm{mg}$ and $5 \mathrm{mg}$ per cent phenformin buffer solutions, compared to that with barbitone buffer alone. This fibrinolytic enhancement by phenformin was found to be logarithmically related to its concentration.

\section{Discussion}

Phenformin is well recognised for its hypoglycaemic and plasma lipid lowering effects in diabetes $[15,16,17,18,19,20,21,22]$. The exact mechanism
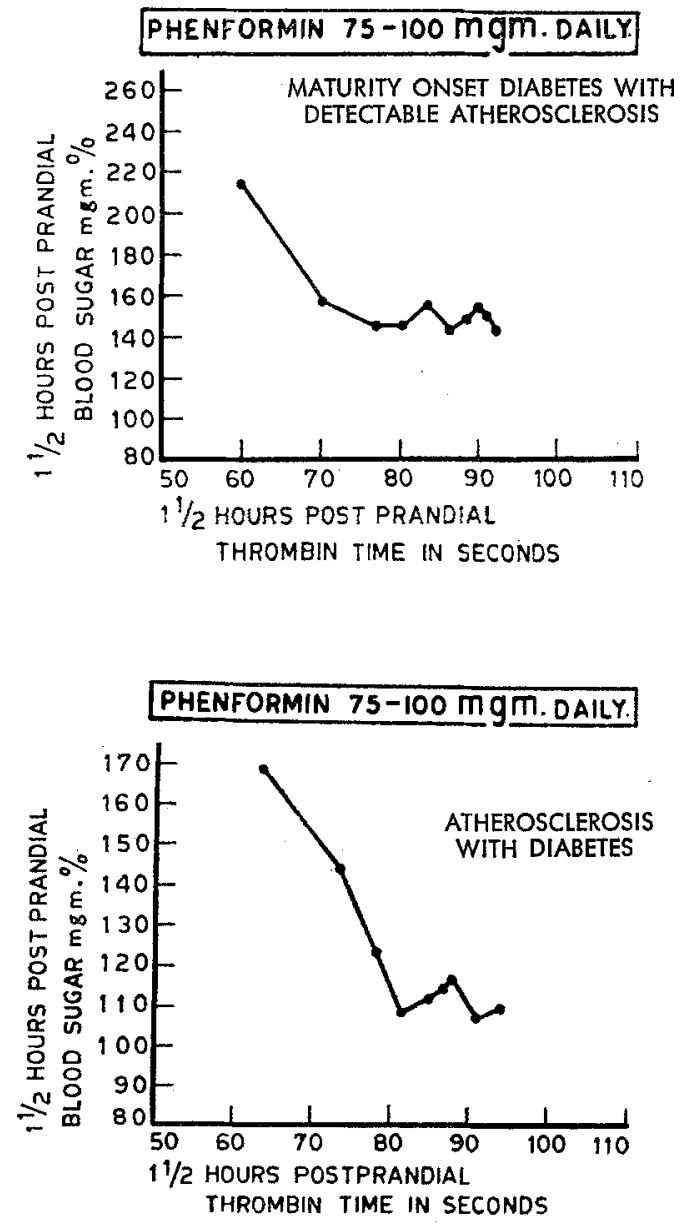

Fig. 2. Correlations between thrombin time and $1 \frac{1 / 2}{\mathrm{hr}}$ post prandial blood sugar response to phenformin therapy in four groups of maturity onset diabetes and atherosclerosis 


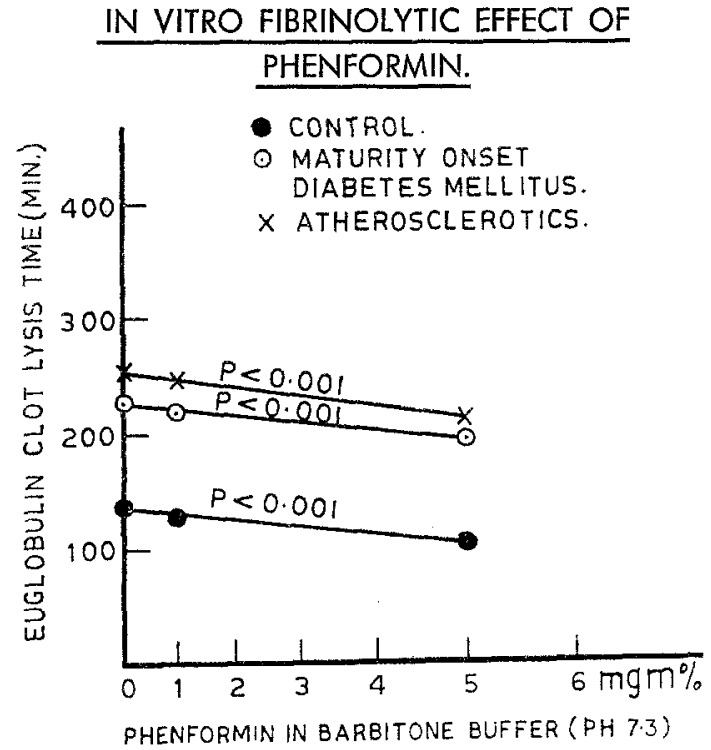

Fig. 3. In vitro fibrinolytic effect of phenformin as shown by mean values of ECLT in normal, diabetic and atherosclerotic plasma, determined with barbitone buffer, $1 \%$ and $5 \%$ phenformin in barbitone buffer solution

of its metabolic effects however, remains uncertain. In recent years, a fibrinolytic property of the drug has also been reported $[6,7,8,9,10]$ and has been suggested to be due to an elevation in the circulating plasminogen activator, secondary to increased production and release in the endothelial cells of the small veins [23].

In the present study, the fibrinolytic property of phenformin was observed in diabetics (groups A, B and D) as well as in non-diabetic atherosclerotics (group C). The study has shown that, besides its fibrinolytic activity, the drug has a highly significant action in prolonging the reduced thrombin time in both diabetes and atherosclerosis. This restoration of the normal thrombin-fibrinogen reaction would reduce the consumption and increase the release of the endothelial plasminogen activator, resulting in an elevation in its plasma level. The fibrinolytic activity of phenformin would thus be considered as secondary to its action in retarding an accelerated thrombinfibrinogen reaction (Fig. 4).

The results of the in vitro studies, on the other hand, provide evidence of a chemical fibrinolytic activity of phenformin, unequivocally observed in normal, diabetic and atherosclerotic plasma. This chemical property of the drug was hitherto unknown.

The results therefore suggest that phenformin can be described as a true 'antifibrinopathic agent' in that it acts both by retarding the thrombin-fibrinogen reaction and accelerating fibrinolysis.

The blood sugar lowering effect of phenformin was observed to be directly related to its action in prolonging reduced thrombin time in the two disorders (Fig. 2). The hypoglycaemic effect of the drug was, however, found to be limited to the restoration of the normal thrombin time; thereafter, no further reduction in blood sugar occurred despite continued phenformin therapy. This observation suggests that the hypoglycaemic effect of phenformin is secondary to its antifibrin action and is due to an increased plasma insulin clearance brought about by the dissolution of the microvascular barrier (Fig. 4). This would also explain the observations of earlier workers that phenformin acts by facilitating plasma insulin clearance rather than by increasing glucose uptake by the tissue $[25,26]$ and that phenformin lowers the plasma insulin level in diabetes $[24,27,28]$.

The antifibrin component of its pharmacological action would also explain the therapeutic limitations of phenformin in the control of long standing diabetics and in normalising the reduced fibrinolytic state when given alone. While enhancing plasma insulin clearance by dissolution of the primary fibrin lesion, the drug may have no effect on the secondary hyperplastic changes in the intima or basement membrane of the microvessels. In the present study, this limitation of phenformin is clearly brought out by the relatively poorer hypoglycaemic response in the established cases of diabetes (Groups A and B) than in the recently detected or non-diabetic subjects (Groups $\mathrm{C}$ and $\mathrm{D}$ ); the blood sugar fall ranged from $150-$ $122.3 \mathrm{mg}$ per $100 \mathrm{ml}$ and 107 to $87.4 \mathrm{mg}$ per $100 \mathrm{ml}$ in the two groups (Fig. 1).

The limitation of phenformin in redressing the reduced fibrinolytic state in diabetic and atherosclerotic subjects has been confirmed in the present observation that even after six months of therapy, the ECLT values remained significantly higher than normal (Table 1). Studies of ECLT response to phenformin therapy by earlier workers showed a state of resistance after a period of $3-4$ months of treatment, which could be overcome by a supplementary anabolic agent $[6,7,8,9,10]$. This "Phenformin resistance", appears to be related to the attainment of normal thrombin time and is similar to its limited hypoglycaemic effect in diabetes. The reported augmentation of fibrinolytic activity by an anabolic steroid might be due to a regenerative action on the secondary intimal pathology.

The present study has confirmed the lipid lowering effects and the clinical benefit of phenformin in diabetes and in atherosclerotic subjects with or without diabetes. Absence of fresh cardiovascular complications and remarkable improvement in anginal symptoms observed during phenformin therapy can 


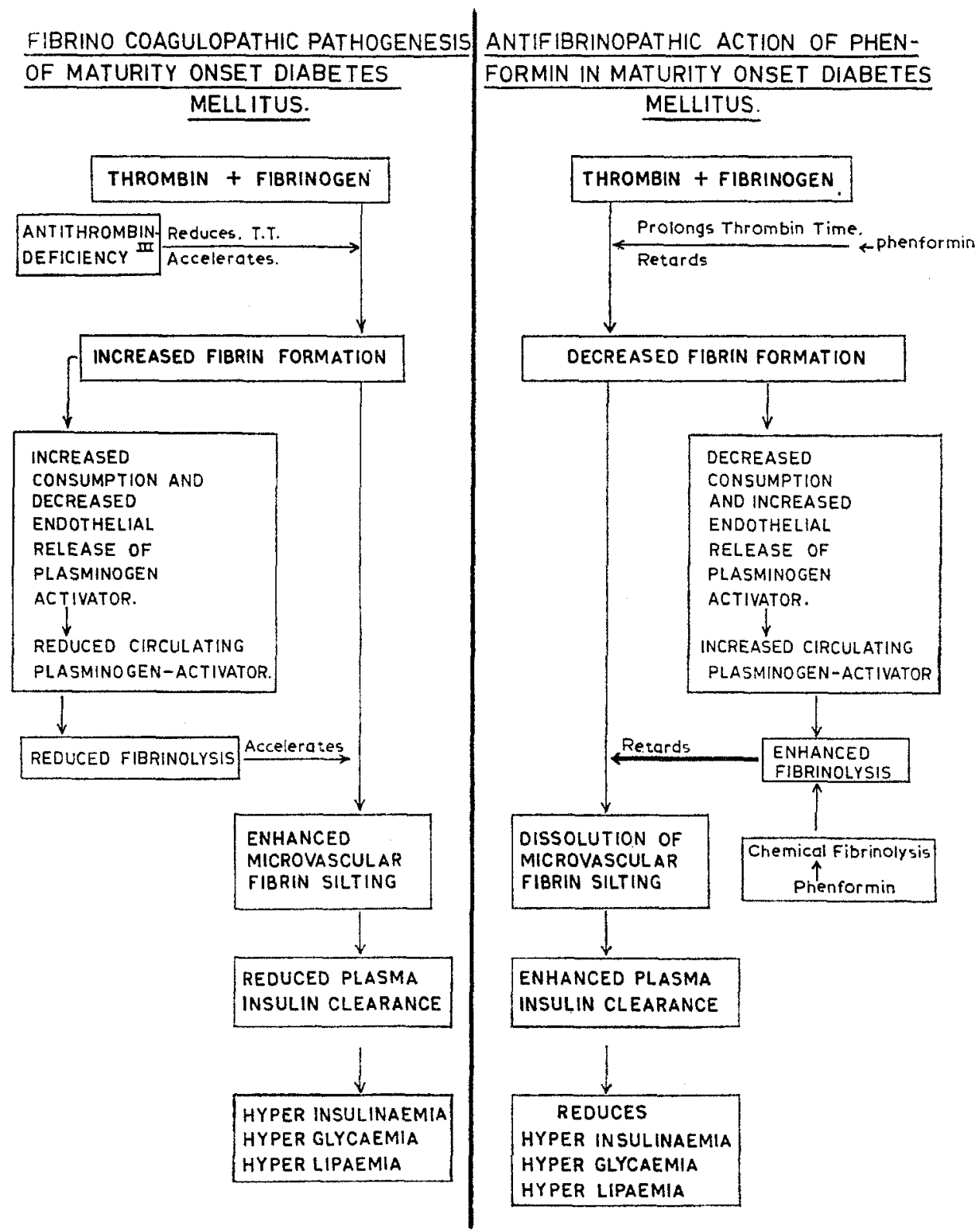

Fig. 4. Hypothesis for fibrinocoagulopathic pathogenesis in maturity onset diabetes mellitus and antifibrinopathic action of phenformin

be explained by its antifibrin action on the macrovascular lesions in atherosclerosis.

The study therefore establishes the antifibrin action of phenformin and lends support to the hypothesis of a primary "fibrinopathic" pathogenesis of maturity onset diabetes mellitus and atherosclerosis ${ }^{3}$.

Acknowledgement. We are grateful to Brig. S. Gopalakrishnan, MC, Medical Superintendent for his kind permission to publish this work. We are thankful to Dr. A. D. Taskar, Chief of Biostatistic Division of I.C.M.R. and his colleagues for their kind cooperation in carrying out the statistical analysis of the present work. The work was carried out with the financial grants received from Research and Development Organisation of Ministry of Defence, Government of India.

\section{References}

1. Banerjee, R. N., Sahni, A. L., Kumar, V.: Plasma thrombin clotting time and plasma fibrinogen in diabetes mellitus and atherosclerosis. Indian J. med. Res. 60, 1432-1442 (1972)

2. Banerjee, R. N., Sahni, A. L., Kumar, V.: Fibrinocoagulopathy in maturity onset diabetes mellitus and atherosclerosis, p. 316. The International Society on Thrombosis 
and Haemostasis. Abstracts, III congress, Washington, D. C. August, 22-26, 1972

3. Banerjee, R. N., Sahni, A. L., Kumar, V.: Fibrinocoagulopathy in maturity onset diabetes mellitus and atherosclerosis. Thrombosis et diath., Haemorrhagica (Stuttg.) 30, 123-132 (1973)

4. Banerjee, R. N., Kumar, V., Sahni, A. L., Arya, M.: Antithrombin III deficiency in maturity onset diabetes mellitus and atherosclerosis. Thrombosis et diath., Haemorrhagica (Stuttg.) 31, 153-159 (1974)

5. Banerjee, R. N., Rao, S. R., Bardhan, J.: Experimental production of diabetes mellitus by non-endocrinal approach. Nature 251, No. 5470, 51-52 (1974)

6. Fearnley, G. R., Chakrabarti, R.: Pharmacological enhancement of fibrinolytic activity of blood. J. clin. Path. 17, 328-332 (1964)

7. Fearnley, G. R., Chakrabarti, R., Hocking, E. D.: Fibrinolytic effects of diguanides plus ethyloestrenol in occlusive vascular disease. Lancet 1967 II, 1008-1011

8. Fearnley, G. R., Chakrabarti, R., Evans, J. F.: Fibrinolytic and defibrinating effect of phenformin plus ethyloestrenol in vivo. Lancet 1969 I, 910-914

9. Menon, I. S., Cunliffe, W. J., Weightman, D., Dewar, H. A.: Phenformin and stanazolol in blood fibrinolytic activity. Brit. med. J. 1970 I, 428

10. Davidson, J. F., Lochhead, M., McDonald, G. A., Mc Nicol, G. P.: Fibrinolytic enhancement by Stanazolol-a double blind trial. Brit. J. Haemat. 22, 543-559 (1972)

11. Fajans, S. S., Conn, J. W.: The early recognition of diabetes mellitus. Ann. N.Y. Acad. Sci. 32, 208-218 (1959)

12. Asatoor, A. M., King, E. J.: Simplified colorimetric blood sugar method. Proceedings of the biochemical society. Biochem. J. 56, XLiv (1954)

13. Van Handel, E., Zilversmit, D. B.: Micro method for the direct determination of serum triglycerides. J. Lab. clin. Med. 50, 152-157 (1957)

14. Von Kaulla, K. N., Schultz., R. L.: Methods for the evaluation of human fibrinolysis. Amer. J. clin. Path. 29, $104-112$ (1958)

15. Ungar, G., Freedman, L., Shapiro, S. L.: Pharmacological studies of a new oral hypoglycaemic drug. Proc. Soc. exp. Biol. (N.Y.) 95, 190-194 (1957)

16. Hall, G. H., Crowley, M. F., Bloom, A.: Oral treatment of diabetes, trial of phenethylbiguanide (D.B.I.). Brit. med. J. 2, 71-74 (1958)

17. Bloom, A., Richards, J. G.: Phenformin as adjuvant oral therapy in diabetes. Brit. med. J., June, 1796-1799 (1961)
18. Fajans, S. S., Moorhouse, J. A., Doorenbos, H., Louis, L. H., Conn, J. W.: Metabolic effects of phenethyl biguanide in normal subjects and in diabetic patients. Diabetes 9, 194-201 (1960)

19. Schwartz, M. J., Mirsky, S., Schaefer, L. E.: The effect of phenformin hydrochloride on serum cholesterol and triglyceride levels of the stable adult diabetic. Metabolism 15, 808-822 (1966)

20. Alterman, S. L., Lopez-Gomez, A. A.: Phenformin effect on body weight, lipids and glucose regulation. Ann. N.Y. Acad. Sci. 148, 884-891 (1968)

21. Mirsky, S.: Influence of hypoglycaemic therapy on blood lipids and body weight in diabetes mellitus. Ann. N.Y. Acad. Sci. 148, 937-944 (1968)

22. Schaefer, L. E.: Hyperlipidaemia. Ann. N.Y. Acad. Sci. 148, 925-936 (1968)

23. Isaacson, S., Nilsson, I. M.: Effect of treatment with combined phenformin and ethyloestrenol on the coagulation and fibrinolytic system. Scand. J. Haemat. 7, 404-408 (1970)

24. Arky, R. A., Abramson, E. A.: Insulin response to glucose in the presence of oral hypoglycaemics. Ann. N.Y. Acad. Sci. 148, 768-777 (1968)

25. Butterfield, W. J. H., Garratt, C. J., Whichelow, M. J.: Peripheral hormone action: studies on the clearance and effect of 131I iodo-insulin in the peripheral tissues of normal, acromegalic and diabetic subjects. Clin. Sci. 24, 331341 (1963)

26. Whichelow, M. J., Butterfield, W. J. H.: In vivo studies in man on the hypoglycaemic action of phenformin. Postgrad. med. J. (May Suppl.) 24, 24-29 (1969)

27. Grodsky, G. M., Karam, J. H., Pavlatos, F., Forsham, P. H.: Reduction by phenformin of excessive insulin levels after glucose loading in obese and diabetic subjects. Metabolism 12, 278-286 (1963)

28. BoshelI, B. R., Roddam, R. F., McAdams, G. L.: Effects of phenformin on insulin reserve and release. Ann. N.Y. Acad. Sci. 148, 756-767 (1968)

Dr. R. N. Banerjee

Dept. of Haematology

and Nuclear Medicine

Safdarjang Hospital

New Delhi

India 